\title{
LRP1 plays a major role in the amyloid- $\beta$ clearance in microglia
}

\author{
Aurelie N'Songo ${ }^{*}$, Takahisa Kanekiyo, Guojun Bu \\ From Molecular Neurodegeneration: Basic biology and disease pathways \\ Cannes, France. 10-12 September 2013
}

\begin{abstract}
Background
Alzheimer's disease (AD), a progressive neurodegenerative disorder and the most prevalent type of dementia in the elderly, is characterized by the accumulation and deposition of amyloid- $\beta$ (A $\beta$ ) peptides and hyperphosphorylated tau in the brain. Impairment of $A \beta$ metabolism induces the formation of toxic $\mathrm{A} \beta$ oligomers as well as the deposition of $A \beta$ in intraneuronal spaces and senile plaques, ultimately resulting in neuronal death. While familial AD is known to be caused by genetic mutations leading to an increase in $A \beta$ production, several lines of evidence suggest that sporadic $\mathrm{AD}$ is due to an impairment of $A \beta$ clearance. $A \beta$ is cleared from the central nervous system by elimination through the blood-brain barrier, extracellular proteolytic degradation or cellular uptake and subsequent lysosomal degradation. The low-density lipoprotein receptor-related protein 1 (LRP1) has been shown to play a major role in $A \beta$ metabolism in neurons, astrocytes and brain vessels. LRP1 is a large transmembrane receptor which mediates endocytosis of more than 30 ligands including apolipoprotein $\mathrm{E}$ and $\alpha$ t2-macroglobulin. Microglia cells are the resident immune and phagocytic cells in the brain and are likely involved in the pathogenesis of $A D$ by contributing to $A \beta$ clearance. Thus, we focused on roles of LRP1 in A $\beta$ clearance in microglia.
\end{abstract}

\section{Materials and methods}

Mouse microglial BV2 cells and primary microglia from wild type C57BL/6 mice were used in this study. Knockdown of LRP1 was performed by transfection with LRP1specific siRNA using Lipofectamine 2000 (Invitrogen), and cells were used for analysis 48 hours after transfection. Control and LRP1-suppressed cells were incubated with fluorescently labeled $A \beta 42$ or microspheres, which are internalized through phagocytosis, and then cellular uptake of these molecules was quantified by FACS after 4 hours of incubation. Furthermore, the cellular localization of fluorescently labeled $A \beta 42$ was assessed using confocal laser microscopy.

\section{Results}

LRP1 is highly expressed in both BV2 cells and primary mouse microglia cells. While microglial cells efficiently internalized A $\beta$, LRP1-suppressed cells showed a decrease of A $\beta 42$ uptake when analyzed by FACS. Consistent with FACS results, we observed less internalized $A \beta$ in LRP1-suppressed microglia cells detected primarily in the lysosomal compartments by confocal microscopy after incubation with $A \beta$ compared to control cells. These results indicate that internalized $A \beta$ is targeted for lysosomal trafficking in the microglia. We also found that the uptake of microspheres was suppressed by the deletion of LRP1 in microglia, suggesting that LRP1 mediates $\mathrm{A} \beta$ phagocytosis and subsequent degradation in microglia.

\section{Conclusion}

Our results indicate that LRP1 plays an important role in cellular uptake of $A \beta$ in microglia. The disturbances of LRP1-mediated A $\beta$ clearance in microglia might be involved in AD pathogenesis.

Published: 13 September 2013

doi:10.1186/1750-1326-8-S1-P33

Cite this article as: N'Songo et al:: LRP1 plays a major role in the amyloid- $\beta$ clearance in microglia. Molecular Neurodegeneration 2013 8(Suppl 1):P33.

Department of Neuroscience, Mayo Clinic, Jacksonville Fl, USA 